\title{
Inhibition of neuroblastoma cell growth by TREX1-mutated human lymphocytes
}

\author{
A. PULLIERO ${ }^{1}$, B. MARENGO ${ }^{2}$, C. DOMENICOTTI ${ }^{3}$, M.G. LONGOBARDI $^{1}$, E. FAZZI $^{4}$,

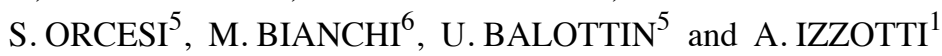 \\ ${ }^{1}$ Department of Health Sciences, University of Genoa, I-16132 Genoa; ${ }^{2}$ Gaslini Institute, I-16145 Genoa; \\ ${ }^{3}$ Department of Experimental Medicine, University of Genoa, I-16132 Genoa; ${ }^{4}$ Department of Mother and Child, Child \\ Neurology and Psychiatry Unit, University of Brescia, I-25123 Brescia; ${ }^{5}$ Department of Child Neurology and Psychiatry, \\ IRCCS 'C. Mondino National Institute of Neurology Foundation', University of Pavia, I-27100 Pavia; ${ }^{6}$ Laboratory of \\ Experimental Neurobiology, IRCCS ‘C. Mondino National Institute of Neurology Foundation’, I-27100 Pavia, Italy
}

Received December 8, 2011; Accepted January 23, 2012

DOI: $10.3892 / o r .2012 .1696$

\begin{abstract}
T lymphocytes play a major role in counteracting cancer occurrence and development. Immune therapies against cancer are focused on eliciting a cytotoxic $\mathrm{T}$ cell response. This anticancer activity is related to a variety of mechanisms including the activation of cytokines and proapoptotic mediators. Interferon $\alpha$ is an established inhibitor of cancer cell growth. A clinical situation involving the coexistence of high interferon $\alpha$ levels and lymphocyte activation is the Aicardi-Goutières syndrome, a progressive encephalopathy arising usually during the first year of life characterized by intracranial basal ganglia calcifications, leukodystrophy and microcephaly. Aicardi-Goutières syndrome 1 mutation silences the TREX1 gene, a major endogenous nuclease. The in vitro study presented herein evaluates the efficacy of the TREX1 mutation in potentiating the anticancer properties of T cells. A TREX1-mutated lymphocyte cell line was derived from an Aicardi-Goutières syndrome patient and co-cultured with neuroblastoma cells and vascular endothelial cells in the presence of interferon $\alpha$. TREX1-mutated lymphocytes exerted marked inhibitory action on neuroblastoma cell growth. Cathepsin D was recognized by qPCR as the main mediator produced by TREX1-mutated lymphocytes involved in the inhibition of neuroblastoma cell growth. These effects were enhanced in the presence of interferon $\alpha$. Similar inhibitory effects in cell growth were exerted by TREX1-mutated lymphocytes towards vascular endothelial cell angiogenesis as evaluated on Matrigel. The results obtained provide evidence that mutations of the TREX1 gene increase the capability of
\end{abstract}

Correspondence to: Professor Alberto Izzotti, Department of Health Sciences, University of Genoa, Via A. Pastore 1, I-16132 Genoa, Italy

E-mail: izzotti@unige.it

Key words: Aicardi-Goutières syndrome, TREX1-mutated lymphocytes, neuroblastoma, angiogenesis, interferon $\alpha$
T-lymphocytes to inhibit growth of neoplastic neuronal cells and related angiogenesis.

\section{Introduction}

T lymphocytes play a major role in counteracting cancer occurrence and development. Cancer-directed immune-based therapies have focused on eliciting a cytotoxic $\mathrm{T}$ cell response, primarily due to the fact that cytotoxic $\mathrm{T}$ cells can directly kill tumors. T helper cells are central to the development of an immune response by activating antigen-specific effector cells and recruiting cells of the innate immune system such as macrophages and mast cells. T helper 1 (Th1) cells, characterized by secretion of interferon $\gamma($ IFN- $\gamma$ ) and tumor necrosis factor $\alpha$ (TNF- $\alpha)$, are primarily responsible for activating and regulating the development and persistence of cytotoxic T cells. In addition, Th1 cells activate antigen-presenting cells and induce the production of a limited type of antibodies that can enhance the uptake of infected cells or tumor cells into antigen-presenting cells (1).

This anticancer activity is related to a variety of mechanisms, including a network of interactions between tumor and immune cells involving cell surface inhibitory receptors and co-stimulatory molecules, intracellular regulatory pathways, immunosuppressive cytokines and proapoptotic mediators, which may operate in concert to orchestrate tumor-immune escape. This emerging pool of inhibitory checkpoints can affect the physiology of innate immune cells, including dendritic cells, macrophages and natural killer cells, as well as different subsets of $\mathrm{T}$ cells, to fine tune their effector function. The synergistic combination of strategies aimed at overcoming regulatory signals and/or stimulating effector pathways, may offer therapeutic advantages as adjuvants of conventional anticancer activity (2). IFN- $\alpha$ is an established inhibitor of cancer cell growth. Interferons (IFNs) are a family of cytokines that exhibit various biological activities. Apart from their role in the immune response, IFNs have been known to modulate cell proliferation and to induce apoptosis (3). This effect is amenable to the antiangiogenic effect of this molecule, whose clinical applications include, in 
addition to cancer therapy, (4) therapy of diseases recognizing blood vessel proliferation as main pathogenic factors, such as diabetic retinopathy (5). A clinical situation characterized by the coexistence of high IFN- $\alpha$ levels and lymphocyte activation in the brain is the Aicardi-Goutières syndrome (AGS), a progressive encephalopathy arising usually during the first year of life and characterized by basal ganglia calcifications, leukodystrophy, and microcephaly (6). Typically, IFN- $\alpha$ levels and lymphocytes are increased in the cerebrospinal fluid of AGS patients in the absence of infections (7). However, IFN- $\alpha$ and CSF lymphocyte levels are within the normal limits following the acute phase of the disease. AGS is a rare genetic disease whose 5 genetic loci have been identified as follows: AGS1 3p21, AGS2 13q14.3, AGS3 11q13.2, AGS4 19q13.13 and AGS5 20q11 (8). Of these mutations, the AGS1 mutation targets the TREX1 gene, and is related to the most aggressive AGS clinical phenotypes, with severe symptoms often arising immediately after birth (9).

In our previous studies we demonstrated that AGS lymphocytes are characterized by profound alterations of gene expression mainly oriented towards IFN- $\alpha$-dependent lymphocyte activation and angiogenesis inhibition $(7,10)$. This situation results in the inhibition of neural cell growth and insufficient development of brain vessels.

Based on these finding, we sought to examine the possibility that AGS mutations enhance the antitumoral activity of $\mathrm{T}$ lymphocytes whenever induced antiangiogenic and antiproliferative activities are directed towards cancer cells. In particular, we hypothesized that the AGS TREX1 mutation is able to potentiate the anticancer properties of $\mathrm{T}$ lymphocytes triggered by IFN- $\alpha$. For this purpose we performed the study presented herein, utilizing co-cultures of normal and AGS-mutated lymphocytes with endothelial and neuroblastoma cells. Endothelial cells were grown on Matrigel plates, thus mimicking the formation of blood vessel, as it occurrs in neoplastic tissues, by the angiogenesis Matrigel assay. Our study aimed to evaluating the role of lymphocyte as effector of interferon-induced angiogenesis inhibition and neurotoxicity and to establish the possible contribution of AGS mutations. We developed an immortalized line of TREX1-mutated lymphocytes providing evidence that the TREX1 mutation is capable of potentiating the anticancer properties of $\mathrm{T}$ cells.

\section{Materials and methods}

Cell cultures. The lymphocytes were isolated from freshly collected whole blood by Histopaque ${ }^{\circledR}-1077$ (Sigma-Aldrich, St. Louis, MO) gradient centrifugation and inserted into co-culture baskets (100,000 cells/basket). Interleukin 2 (4 $\mu \mathrm{g} /$ $\mathrm{ml})$ and phytohemagglutinin $(2 \mu \mathrm{g} / \mathrm{ml})$ were added to the culture medium to selectively induce T-lymphocyte development.

Cell lines derived from wild-type lymphocytes obtained from a matched healthy control (male, 6 years old) and AGS mutated lymphocytes collected from an AGS patient (male, 6 years old) were examined. The AGS patient was identified as a carrier of double AGS1 TREX heterozygous mutation (c.262 ins AG het + c.290 g>a R97H het) (Professor Y. Crow, University of Manchester, UK). The enrolled subject was treated in accordance with the Declaration of Helsinki. This study was approved by the Ethics Committee of the IRCSS
Mondino, University of Pavia, Italy. The Epstein-Barr (EBV) virus was used to convert lymphocytes to continuously dividing, efficiently immortalized lymphoblastoid cell lines.

Transforming virus were obtained from the lymphoblastoid marmoset cell line B95-8 (ECACC). This line is established by infecting marmoset lymphocytes with EBV from a human patient with infectious mononucleosis. Cyclosporine A is used as an immunosuppressive drug to enhance the outgrowth of EBV-infected lymphocytes (11).

Peripheral blood mononuclear cells were resuspended with growth medium, RPMI-1640 (PAA), supplemented with 20\% FBS (PAA), 1\% L-glutamine $200 \mathrm{mM}$ (PAA), $1 \%$ penicillinstreptomycin (PAA) and $5 \mu \mathrm{g} / \mu 1$ of CS-A (Sigma) and the same volume of virus-containing supernatant from B95-8. After 1 week in culture, half of the growth medium was removed and was replaced with fresh medium containing cyclosporine A. After 2-3 weeks in culture small clumps became visible. The cell lines were then stored at $-180^{\circ} \mathrm{C}$ in liquid nitrogen until use. SH-SY5Y, a human neuroblastoma cell line, was cultured in MEM/Ham's F12 medium in presence of $10 \%$ fetal calf serum and glutamine.

Human umbilical endothelial cells (HUVEC) were grown in culture medium M199 supplemented with 20\% FBS, $100 \mathrm{mg} / \mathrm{ml}$ endothelial cell growth supplement (ECGS), $100 \mathrm{mg} / \mathrm{ml}$ heparin, $2 \mathrm{mM} \mathrm{L}$-glutamine, $100 \mathrm{U} / \mathrm{ml}$ penicillin and $100 \mathrm{mg} / \mathrm{ml}$ streptomycin. Cells were maintained at $37^{\circ} \mathrm{C}$ and $5 \% \mathrm{CO}_{2}$ and used for co-culture experiments at the fourth passage.

Evaluation of angiogenesis. Angiogenesis assay was performed on Matrigel (Becton-Dickinson), a crude extract of basement membrane of Engelbreth-Holm-Swarm tumor, mimicking capillary morphogenesis (12). Subsequently, healthy and TREX1mutated lymphocytes were inserted into the central co-culture basket $(100,000$ cells/basket) and exposed to increasing concentrations of IFN- $\alpha(80,1,000$ and 10,000 U/ml), doses selected on the basis of preliminary dose-response toxicity experiments (data not shown). Co-cultures were maintained for 2 days. The lymphocyte basket was then removed and angiogenesis was assessed by observing the development of vessel-like bodies by an inverted microscope equipped with a digital camera (Leica DMIRB and camera DFC 320R2, Leica Microsystems, Wetzlar, Germany). The morphological changes of capillary-like networks were recorded at 24-h intervals. Capillaries were defined as multicellular cords between two cell aggregates.

Evaluation of growth inhibition of neuroblastoma cells by lymphocyte co-culture. SH-SY5Y cells $(100,000$ cells/well) were seeded on co-culture plates coated with Matrigel. Under these conditions neuroblastoma cells were grown, developing reciprocal adherence and emitting filopodia, thus mimicking the formation of neoplastic neural tissue (13). In fact, proliferation of SH-SY5Y human neuroblastoma cells as well as their migration and invasion, create, through an extracellular matrix barrier, a neural network. Subsequently, healthy and TREX1-mutated lymphocytes were inserted into the central co-colture basket and exposed to increasing concentrations of IFN- $\alpha(0,80,1,000,5,000$ and 10,000 U/ml). Co-cultures were maintained for 12 days, then the lymphocytes basket was 
Table I. Primers and probes identified using a molecular Beacon designer software. ${ }^{\mathrm{a}}$

\begin{tabular}{lll}
\hline Gene & & \multicolumn{1}{c}{ Sequence } \\
\hline Cathepsin D & & \\
& Sense & TCTGTCCTACCTGAATGTCACC \\
& Antisense & CCTCACAGCCCTCCTTGC \\
& Beacon sequence & FAM-CGCGATCGGCCACCTCCACCTGGTCCAGGGATCGCG-BHQ1 \\
GAPDH & & \\
& Sense & CAAGGCTGAGAACGGGAAG \\
& Antisense & AAGACGCCAGTGGACTCC \\
& Beacon sequence & FAM-CGCGATCATCTCGCTCCTGGAAGATGGTGATGGATCGCG-BHQ1
\end{tabular}

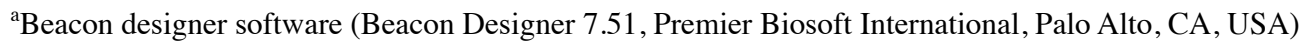

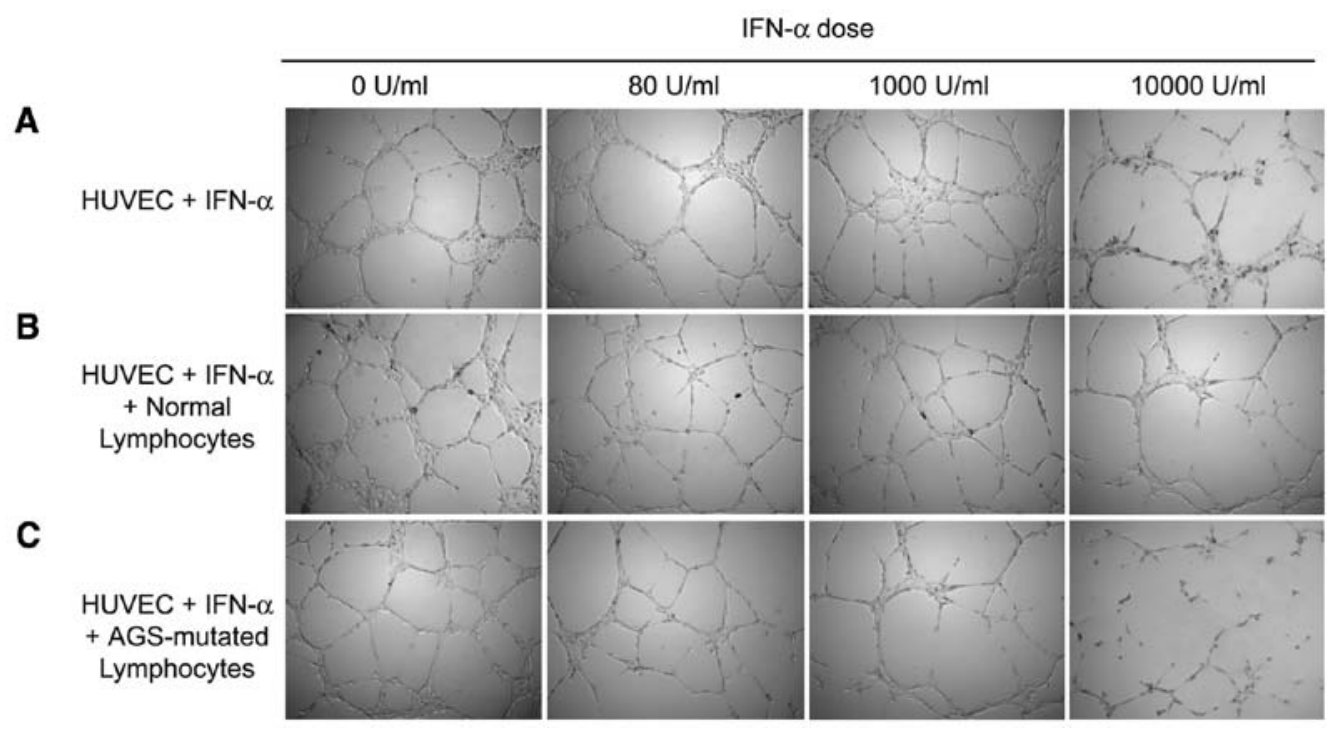

Figure 1. Vessel-like body development in HUVEC cell cultures on Matrigel (A) alone, or in the presence of (B) normal or (C) TREX1-mutated T lymphocytes. Increasing IFN- $\alpha$ doses were added (from left to right, control 0, 80, 1,000 and 10,000 U/ml). Magnification, x5.

removed and the morphology of neuroblastoma (NB) cells was analyzed using an inverted microscope (Leica DM IRB) equipped with a digital camera (Leica DFC 320R2).

Quantitative PCR.Cathepsin D is a potent lymphocyte protease playing an important role in cytotoxicity and neurodegeneration. The role of this protease activity in AGS pathogenesis has been previously documented (7). Expression of the cathepsin D gene was evaluated by real-time quantitative PCR (qPCR) in control and AGS TREX1-mutated lymphocytes exposed to increasing IFN- $\alpha$ concentrations.

Total RNA was extracted by TRIzol (Invitrogen Corp., Carlsbad, CA, USA), and its purity and integrity was evaluated by a fiber optic spectrophotometer (NanoDrop Thermo Fisher Scientific, Wilmington, DE, USA) and by capillary electrophoresis (Bioanalyzer, Agilent Technologies, Inc., Santa Clara, CA, USA), respectively.

Total RNA (50 ng) was added to $0.5 \mu \mathrm{g}$ oligo(dT) and $10 \mathrm{mM}$ dNTP mix $(1 \mu \mathrm{l})$ and water to a $12-\mu \mathrm{l}$ final reaction volume, that was then incubated at $65^{\circ} \mathrm{C}$ for $5 \mathrm{~min}$. Subsequently, $5 \mathrm{X}$ first strand buffer (4 $\mu \mathrm{l}), 0.1$ M DTT $(2 \mu \mathrm{l})$, RNAse OUT $(1 \mu \mathrm{l})$, DNA reverse transcriptase superscript II (1 $\mu \mathrm{l}$; Invitrogen) were added and incubated for $60 \mathrm{~min}$ at $42^{\circ} \mathrm{C}$ followed by $15 \mathrm{~min}$ at $70^{\circ} \mathrm{C}$ to stop the reaction. Finally, $2 \mu \mathrm{l}$ of the obtained cDNA mix were added to a $200-\mu 1$ vessel containing $10 \mathrm{X}$ PCR buffer (5 $\mu \mathrm{l}), 50 \mathrm{mM} \mathrm{MgCl} 2(2 \mu \mathrm{l}), 100 \mathrm{mM}$ dNTP mix $(0.4 \mu \mathrm{l})$, primer A $10 \mu \mathrm{M}(1 \mu \mathrm{l})$, primer S $10 \mu \mathrm{M}(1 \mu \mathrm{l})$, Platinum ${ }^{\circledR}$ TaqDNA polymerase $(0.5 \mu \mathrm{l}$; Invitrogen) and $10 \mu \mathrm{M}$ specific molecular beacon $(2 \mu \mathrm{l})$. The reaction was carried out in a rotating thermocycler (Rotor-Gene 3000, Corbett Research, Mortlake, Australia) using the following program: $95^{\circ} \mathrm{C}$ for $2 \mathrm{~min} ; 40$ cycles at $94^{\circ} \mathrm{C}$ for $45 \mathrm{sec}, 24^{\circ} \mathrm{C}$ for $30 \mathrm{sec}, 72^{\circ} \mathrm{C}$ for $30 \mathrm{sec}$. A second parallel qPCR reaction was performed using primers and probes specific for the housekeeping gene GAPDH normalizing the cathepsin D expression data for each sample to the relative GAPDH data. Primers and probes used were identified using a molecular Beacon designer software (Beacon Designer 7.51, Premier Biosoft International) (Table I). Statistical analyses were executed using Statview software (Abacus Concept, Berkeley, CA, USA). 


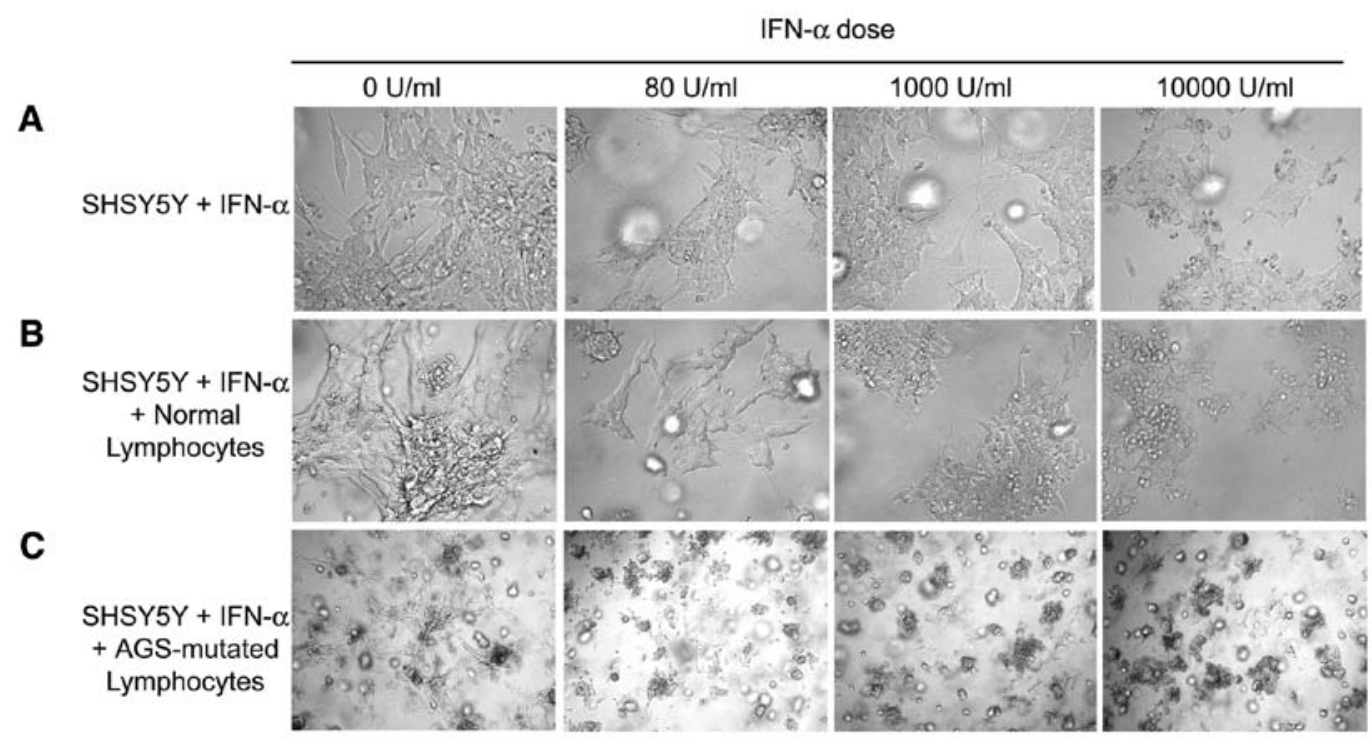

Figure 2. Growth of neuroblastoma SH-SY5Y cells on Matrigel either (A) alone, or in the presence of (B) normal, or (C) TREX1 mutated T lymphocytes. Increasing IFN- $\alpha$ doses were added (from left to right control 0, 80, 1,000 and 10,000 U/ml). Magnification, x20.

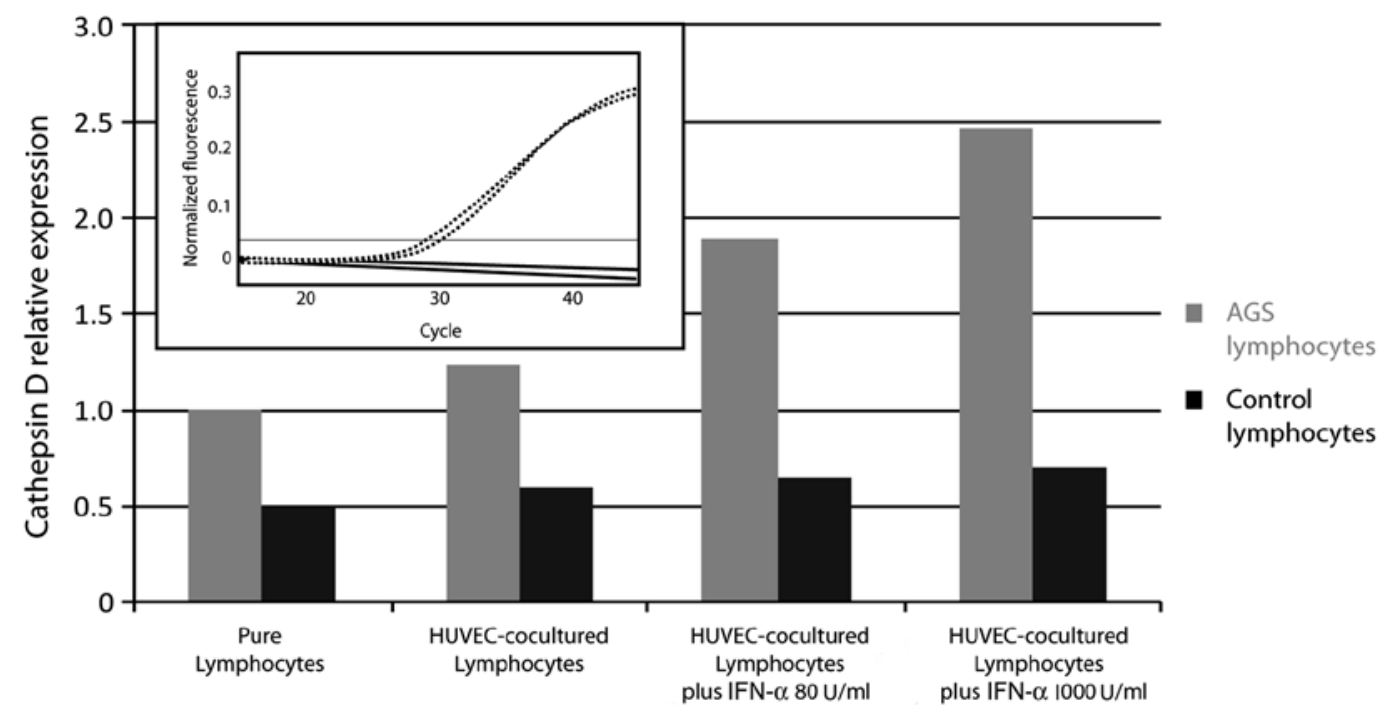

Figure 3. Expression of the cathepsin D gene was evaluated by qPCR in normal (black columns) or TREX1-mutated T lymphocytes (gray columns), in co-culture with HUVEC and in the presence of IFN- $\alpha$ (from left to right). The inset illustrates the qPCR amplification cycles as detected in untreated T lymphocytes, either in the absence (dashed lines) or the presence of TREX1 mutation (continuous lines). The horizontal line in the inset indicates the positivity threshold.

\section{Results}

Antiangiogenic properties of lymphocytes are affected by $I F N-\alpha$ and TREXI mutation. IFN- $\alpha$, when used alone in HUVEC cells, did not inhibit vessel-like bodies formation at 80 and $1,000 \mathrm{U} / \mathrm{ml}$. However, a weak inhibitory effect was observed only at 10,000 U/ml (Fig. 1A). Conversely, the presence of healthy lymphocytes in the co-culture dramatically increased the antiangiogenic properties of IFN- $\alpha$ when administered at 1,000 and $10,000 \mathrm{U} / \mathrm{ml}$, while a limited effect was observed at $0 \mathrm{U} / \mathrm{ml}$ (IFN- $\alpha$ absence) or $80 \mathrm{U} / \mathrm{ml}$ (Fig. 1B). As shown in Fig. 1C, the presence of AGS TREX1-mutated lymphocytes increased the antiangiogenic effect of IFN- $\alpha$ at all tested doses (Fig. 1C).
Antiproliferative properties of lymphocytes are affected by $I F N-\alpha$ and TREXI mutation. The effects of lymphocytes were also assessed in SH-SY5Y cells, which normally grow onto Matrigel emitting filopodia, thus developing a tissue web-like aspect (Fig. 2A). After 12 days, control cells, in the absence of any treatment, reached confluence and completely covered the plate with massive filopodia emissions (data not shown).

IFN- $\alpha$, when used alone, did not inhibit the monolayer growth of neuroblastoma cells at 80 and $1,000 \mathrm{U} / \mathrm{ml}$. A weak inhibitory effect was observed only at $10,000 \mathrm{U} / \mathrm{ml}$, resulting in a decrease of filopodia formation and cell interconnections (Fig. 2A). Conversely, the presence of normal lymphocytes in the co-culture dramatically increased IFN- $\alpha$-induced effects 
at 1,000 and 10,000 U/ml while a limited effect was observed at 0 and $80 \mathrm{U} / \mathrm{ml}$ (Fig. 2B). Moreover, as shown in Fig. 2C, the presence of AGS TREX1-mutated lymphocytes increased, the effect exerted by IFN- $\alpha$ at all doses resulting in the inhibition of cell growth, loss of filopodia formation, changes in cell shape with the assumption of a spheroid aspect, lack of formation of cell interconnections and a web-like aspect.

Differential effect of IFN- $\alpha$ on cathepsin D expression in wildtype and TREX1-mutated lymphocytes. qPCR results indicate that cathpesin D expression is doubled under basal conditions in AGS TREX1-mutated lymphocytes as compared to healthy lymphocytes (Fig. 3). This difference increased when lymphocytes were co-cultured with HUVEC cells either in the absence or presence of increasing concentrations of IFN- $\alpha$. However, healthy lymphocytes only moderately increased cathepsin D expression in response to IFN- $\alpha$ treatment. Conversely, AGS TREX1-mutated lymphocytes reacted to IFN- $\alpha$ exposure by increasing (1.34-fold) cathepsin D expression both at a low $(80 \mathrm{U} / \mathrm{ml})$ and a high dose $(1,000 \mathrm{U} / \mathrm{ml})$ (Fig. 3).

\section{Discussion}

Apart from their roles in the immune response, IFNs have been known to modulate cell proliferation and to induce apoptosis. Results from the present study provide evidence that lymphocytes are pivotal mediators of IFN- $\alpha$ effects, both in terms of angiogenic potential and neuroblastoma cell growth inhibition. Furthermore, evidence is provided that AGS mutation increases the ability of lymphocytes to exert both of these effects.

IFN- $\alpha$, when used alone, negatively affects the formation of vessel-like bodies by HUVEC cells only at the highestexamined dose. The antiangiogenic properties of IFN- $\alpha$ have been established both in vitro (14) and in vivo (15). However, in vivo effects appear to be more potent than those observed under in vitro conditions (14). The involvement of lymphocytes in the antiangiogenic effect exerted by IFN- $\alpha$ is likely to have a causal role in the potent antiangiogenic effect of IFN- $\alpha$ under in vivo conditions (15). Activated lymphocytes, when used alone, are moderate inhibitors of tumor angiogenesis (16). Conversely, during inflammation, lymphocytes may exert some proangiogenic activities (17).

Our research group has demonstrated that AGS lymphocytes are characterized by profound alterations of gene expression mainly oriented towards the activation of lymphocytes and the inhibition of angiogenesis $(7,10)$.

Based on our findings, the synergism between lymphocytes and IFN- $\alpha$ seems to be required to trigger the antiangiogenic potential of these two anticancer factors. In particular, our results suggest that IFN- $\alpha$ could be used to potentiate the antiangiogenic effect of activated lymphocytes, limiting the formation of novel vessels that support the growth of the neoplastic mass. This finding bears relevance for cancer prevention and therapy with particular reference for the use of activated lymphocytes against cancer development. Of note, a vaccine against prostate cancer has been developed based on the use of activated lymphocytes (18).

The antiangiogenic effect exerted by the IFN- $\alpha /$ lymphocyte synergism is paralleled by the activation of the proteases capable of interfering with cell growth. In fact, the growth of SH-SY5Y cells was inhibited by co-culture with lymphocytes in the presence of IFN- $\alpha$. In this case, lymphocytes induced inhibition of cell growth. These effects are likely mediated by the increased expression of cathepsin D, as documented by the strong parallelism between the level of expression of this gene and the inhibitory effects exerted by lymphocytes on neuroblastoma cell growth. Cathepsin D is a protease detectable in different cell types, whose primary function is to degrade proteins by lysosome proteolysis. In addition, this enzyme is able to induce intercellular matrix digestion, (19) cell membrane damage, (20) and apoptosis (21). Moreover, cathepsin D has been suggested to be involved in Alzheimer's disease, which is in line with increasing evidence that disturbance of the cathepsin homeostasis may contribute to neurodegeneration (19).

Accordingly, abnormally high expression of cathepsin D results in the induction of degeneration in the brain cortex and the central nervous systems. In fact, cathepsin D is considered a main effector of several types of dementia including, Parkinson's disease (22).

Conversely, in tumoral tissue composed of proliferating cells, cathepsin D overexpression plays a role in the development of neoplastic mass cancer appearance (20). Indeed, cathepsin D plays an essential role in the multiple steps of tumor progression, in stimulating cancer cell proliferation, fibroblast outgrowth and angiogenesis, as well as in inhibiting apoptosis. Mutated cathepsin D, devoid of the catalytic activity, still proved to be mitogenic for cancer, endothelial and fibroblastic cells, suggesting an extracellular mode of action of cathepsin D involving the triggering, either directly or indirectly, of a cell surface receptor. Cathepsin D is also a key mediator of apoptosis and its proteolytic activity has been implicated generally in this action (23).

Accordingly, when expressed by immune cells, cathepsin D plays a role in inducing cancer cell death. Our findings that the synergism between IFN- $\alpha$ and T lymphocytes is a potent inducer of cathepsin D expression resulting in the inhibition of neuroblastoma cell growth, bears relevance for cancer therapy. Furthermore, in our experimental conditions, this protective effect is increased by the parallel inhibition of vessel growth supporting cancer development.

These two anticancer effects are increased by the occurrence of silencing mutations in the AGS loci. In particular, examined AGS1 mutations silenced the TREX1 gene, which is a pivotal intracellular nuclease. TREX1-deficiency has been shown to result in intracellular accumulation of single-strand DNA (24), leading to immunological induction (25). Thus, in TREX1-defective cells accumulation of intracellular RNA/ DNA replication intermediates triggers an inappropriate virallike innate immune response (26). TREX1 enzyme deficiency in the AGS has shown that AGS1 cells exhibit chronic ATM (ataxia telangiectasia mutated)-dependent checkpoint activation and TREX1-deficient cells accumulate ssDNA fragments (27).

Other studies have reported the existence of TLR-independent pathways that are activated in response to microbial and host nucleic acids (28) and have shown that TREX1-null mice, which mimic the AGS1 mutation, accumulate IFN- $\alpha$ following activation of a TLR-independent pathway involving the interferon regulatory factor 3 . 
Therefore, the data indicate that TREX1 mutation is a potent endogenous inducer of the innate immune response and an efficacious activator of the TLR pathways. This situation sensitize $\mathrm{T}$ lymphocytes to the IFN- $\alpha$-dependent activation resulting in a dramatic enhancement of their inhibitory effects on cell growth. As documented by the results presented herein, the inhibition of neuroblastoma cell growth is induced both by antiangiogenic effects and cathepsin D overproduction. These findings indicate that TREX-1 mutation is a potent mechanism for increasing the antineoplastic properties of $\mathrm{T}$ lymphocytes. These effects were further enhanced when cells were exposed to low doses of IFN- $\alpha$. This situation has dramatic consequences in AGS patients. In particular, the development of the brain tissue of these newborns was impaired due to inhibition of both vessel and nerve tissue growths $(29,30)$.

However, the same mechanisms, i.e., TREX1 inhibition and IFN- $\alpha$ production, activating T lymphocytes in AGS patients could be beneficial for increasing the ability of T-lymphocytes to counteract the growth of neoplastic cells, as mentioned for neuroblastoma cells; this is further supported by the finding that so far, no cancer, and, in particular, no pediatric neuroblastoma cases have been reported among AGS patients (31).

In conclusion, our data, although carried out using TREX1mutated lymphocytes obtained from one patient, provide evidence that IFN- $\alpha$ and TREX1-mutated lymphocytes are potent inhibitor of angiogenesis and neoplastic cell growth, suggesting that the use of IFN- $\alpha$-activated T-lymphocytes, bearing the TREX1 mutation, could be a novel possible therapeutic strategy to counteract neuroblastoma development.

\section{Acknowledgements}

We thank Professor Y. Crow, University of Manchester, UK for the AGS mutation analysis. This study was supported by the Italian Association for Cancer Research (grant no. 8909) and by the International Aicardi-Goutières Syndrome Association.

\section{References}

1. Knutson KL and Disis ML: Tumor antigen-specific T helper cells in cancer immunity and immunotherapy. Cancer Immunol Immunother 54: 721-728, 2005.

2. Zwirner NW, Croci DO, Domaica CI and Rabinovich GA: Overcoming the hurdles of tumor immunity by targeting regulatory pathways in innate and adaptive immune cells. Curr Pharm Des 16: 255-267, 2010.

3. Ota K, Matsumiya T, Sakuraba H, et al: Interferon- $\alpha 2 b$ induces $\mathrm{p} 21^{\text {cipl/wafl } 1}$ degradation and cell proliferation in HeLa cells. Cell Cycle 9: 131-139, 2010.

4. Burchert A, Müller MC, Kostrewa P, et al: Sustained molecular response with interferon $\alpha$ maintenance after induction therapy with imatinib plus interferon $\alpha$ in patients with chronic myeloid leukemia. J Clin Oncol 28: 1429-1435, 2010.

5. Panetta JD and Gilani N: Interferon-induced retinopathy and its risk in patients with diabetes and hypertension undergoing treatment for chronic hepatitis $\mathrm{C}$ virus infection. Aliment Pharmacol Ther 15: 597-602, 2009.

6. Lanzi G, D'Arrigo S, Drumbl G, Uggetti C and Fazzi E: Aicardi-Goutières syndrome: differential diagnosis and aetiopathogenesis. Funct Neurol 18: 71-75, 2003

7. Izzotti A, Pulliero A, Orcesi S, et al: Interferon-related transcriptome alterations in the cerebrospinal fluid cells of Aicardi-Goutières patients. Brain Pathol 19: 650-660, 2009.
8. Rice GI, Bond J, Asipu A, Brunette RL, Manfield IW, et al: Mutations involved in Aicardi-Goutières syndrome implicate SAMHD1 as regulator of the innate immune response. Nat Genet 41: 829-832, 2009.

9. Rice G, Newman WG, Dean J, et al: Heterozygous mutations in TREX1 cause familial chilblain lupus and dominant AicardiGoutières syndrome. Am J Hum Genet 80: 811-815, 2007.

10. Izzotti A, Fazzi E, Orcesi S, et al: Brain damage as detected by cDNA-microarray in the spinal fluid of patients with AicardiGoutières syndrome. Neurology 71: 610-612, 2008.

11. Ling PD and Huls HM: Isolation and immortalization of lymphocytes. Curr Protoc Mol Biol 28: Unit 28.2, 2005.

12. Ferrari N, Pfeffer U, Dell'Eva R, et al: The transforming growth factor- $\beta$ family members bone morphogenetic protein- 2 and macrophage inhibitory cytokine-1 as mediators of the antiangiogenic activity of $N$-(4-hydroxyphenyl)retinamide. Clin Cancer Res 11: 4610-4619, 2005.

13. Voigt A and Zintl F: Effects of retinoic acid on proliferation, apoptosis, cytotoxicity, migration, and invasion of neuroblastoma cells. Med Pediatr Oncol 40: 205-213, 2003.

14. Abushahba W, Balan M, Castaneda I, et al: Antitumor activity of type I and type III interferons in BNL hepatoma model. Cancer Immunol Immunother 59: 1059-1071, 2010.

15. Zhu Y, Tibensky I, Schmidt J, et al: Interferon-alpha in combination with chemotherapy has potent antiangiogenic properties in an orthotopic mouse model for pancreatic adenocarcinoma. J Immunother 31: 28-33, 2008.

16. Kubota T, Taiyoh H, Matsumura A, et al: Gene transfer of $\mathrm{NK} 4$, an angiogenesis inhibitor, induces CT26 tumor regression via tumor-specific T lymphocyte activation. Int J Cancer 125: 2879-2886, 2009.

17. Murdoch C, Tazzyman S, Webster S and Lewis CE: Expression of Tie- 2 by human monocytes and their responses to angiopoietin-2. J Immunol 178: 7405-7411, 2007.

18. Anderson MJ, Shafer-Weaver K, Greenberg NM and Hurwitz AA: Tolerization of tumor-specific $\mathrm{T}$ cells despite efficient initial priming in a primary murine model of prostate cancer. J Immunol 178: 1268-1276, 2007.

19. Nakanishi $\mathrm{H}$ : Neuronal and microglial cathepsins in aging and age-related diseases. Aging Res Rev 2: 367-381, 2003.

20. Beaujouin M and Liaudet-Coopman E: Cathepsin D overexpressed by cancer cells can enhance apoptosis-dependent chemo-sensitivity independently of its catalytic activity. Adv Exp Med Biol 617: 453-461, 2008.

21. Zheng X, Chu F, Mirkin BL, et al: Role of the proteolytic hierarchy between cathepsin L, cathepsin D and caspase-3 in regulation of cellular susceptibility to apoptosis and autophagy. Biochim Biophys Acta 1783: 2294-2300, 2008.

22. Liaudet-Coopman E, Beaujouin M, Derocq D, et al: Cathepsin D: newly discovered functions of a long-standing aspartic protease in cancer and apoptosis. Cancer Lett 237: 167-179, 2006.

23. Qiao L, Hamamichi S, Caldwell KA, et al: Lysosomal enzyme cathepsin D protects against alpha-synuclein aggregation and toxicity. Mol Brain 21: 1-17, 2008.

24. Yang YG, Lindahl T and Barnes DE: Trex1 exonuclease degrades ssDNA to prevent chronic checkpoint activation and autoimmune disease. Cell 131: 836-838, 2007.

25. Ishii KJ and Akira S: Innate immune recognition of nucleic acids: beyond toll-like receptors. Int J Cancer 117: 517-523, 2005.

26. Lehtinen DA, Harvey S, Mulcahy MJ, Hollis T and Perrino FW: The TREX1 double-stranded DNA degradation activity is defective in dominant mutations associated with autoimmune disease. Biol Chem 283: 31649-31656, 2008.

27. Lindahl T, Barnes DE, Yang YG and Robins P: Biochemical properties of mammalian TREX1 and its association with DNA replication and inherited inflammatory disease. Arthritis Rheum 37: 535-538, 2009.

28. Wagner H: Endogenous TLR ligands and autoimmunity. Adv Immunol 91: 159-173, 2006.

29. Stetson DB, Ko JS, Heidmann T and Medzhitov R: Trex1 prevents cell-intrinsic initiation of autoimmunity. Cell 134: 587-598, 2008.

30. Ramantani G, Kohlhase J, Hertzberg C, et al: Expanding the phenotypic spectrum of lupus erythematosus in AicardiGoutières syndrome. Arthritis Rheum 62: 1469-1477, 2010.

31. Orcesi S, La Piana R and Fazzi E: Aicardi-Goutières syndrome. Br Med Bull 89: 183-201, 2009. 